\title{
BUILDING MECHANICS
}

DOI 10.36622/VSTU.2021.51.3.009

UDC 624.04:531.391.3

\author{
M. N. Kirsanov ${ }^{1}$
}

\section{SPECTRUM OF OWN FREQUENCIES OF A SPATIAL SURFACING GIRDER*}

\author{
«MEI» National Research University ${ }^{1}$ \\ Russia, Moscow
}

${ }^{1}$ D. Sc. in Physics and Mathematics, Prof. of the Dept. of Robotics, Mechatronics,
Dynamics and Strength of Machines, tel.: +7 (495) 362-73-14; e-mail: c216@ya.ru

Statement of the problem. The scheme of a statically definable girder of a spatial rectangular surfacing is discussed. The problem is to identify the formula for the dependence of the lower estimate of the first frequency of the natural oscillations of the structure by means of the Donkerley method on the number of panels. The truss has supports on the sides and consists of separate rod cells connected in pyramids.

Results. Based on the analysis of the sequence of analytical solutions for the first frequency of girders with a different number of panels by induction, the coefficients in the desired formula are derived. The common members of the sequences of coefficients are found as solutions of homogeneous recurrent equations formed according to the results of the calculations using Maple operators. The resulting dependences are obtained in the form of polynomials by the number of panels. A comparison of the analytical solution with the numerical one is provided.

Conclusions. An algorithm for deriving an analytical estimate of the fundamental frequency of oscillations of a spatial structure depending on the number of panels, mass, size, and elastic properties of the material is shown. The spectrum of oscillation frequencies of the structure is analyzed. The resulting dependences can be employed in seismic and structural optimization problems.

Keywords: spatial girder, natural frequency, Donkerley method, lower estimate, induction, Maple, analytical solution, natural frequency spectrum, multiple frequencies.

Introduction. The calculation of the frequencies of natural vibrations of building structures in practice is commonly performed in standard packages by means of the finite element method $[4-6,22]$. The values of natural frequencies are crucial in analyzing the seismic resistance of structures $[11,20,21]$ and under operating conditions under external dynamic influences $[16,18]$.

\footnotetext{
* The research is funded by the Interdisciplinary Scientific and Educational School of Moscow University "Fundamental and Applied Space Research".
}

(C) Kirsanov M. N., 2021 
An alternative to numerical methods for calculating the vibration frequency spectra of structures is the analytical method. What makes the study timely and relevant is that for systems with a large number of degrees of freedom, it is generally impossible to obtain an exact solution to the problem of identifying the natural frequencies in the general case. An exception is the approximate solution of the problem of the lower estimate of the first frequency using the Donkerley method [14]. Among the known solutions, it is possible to single out those for regular (periodic) statically definable rod systems.

R. G. Hutchinson and N. A. Fleck [8, 9] looked at the problem of regular bar structures. There are known analytical solutions for deflection of flat trusses depending on the number of panels $[1-3,10,17,19,23-26]$ obtained by means of the induction method in the Maple mathematical software. The problems of oscillation of flat regular trusses in analytical form were addressed in $[12,15]$.

In this study, the derivation of the formula for the dependence of the first frequency on the number of panels is shown for the model of a statically definable truss of spatial coverage [13]. The spectra of eigenfrequencies of trusses with different numbers of panels are analyzed numerically and some of their features are identified.

1. Surfacing structure. A $2 n a \times 2 n a$ square girder consists of $n^{2}$ individual cells of rods connected in pyramids (Fig. 1). The side length of one cell is $2 a$, the height is $h$ (Fig. 2). Vertical hinged support rods are positioned along the entire perimeter of the structure. The corner joint $A$ is also secured by two additional horizontal braces so that, together with the vertical bar, a spherical joint is formed at point $A$. The corner joint $B$ is secured by vertical and horizontal rods (cylindrical joint).

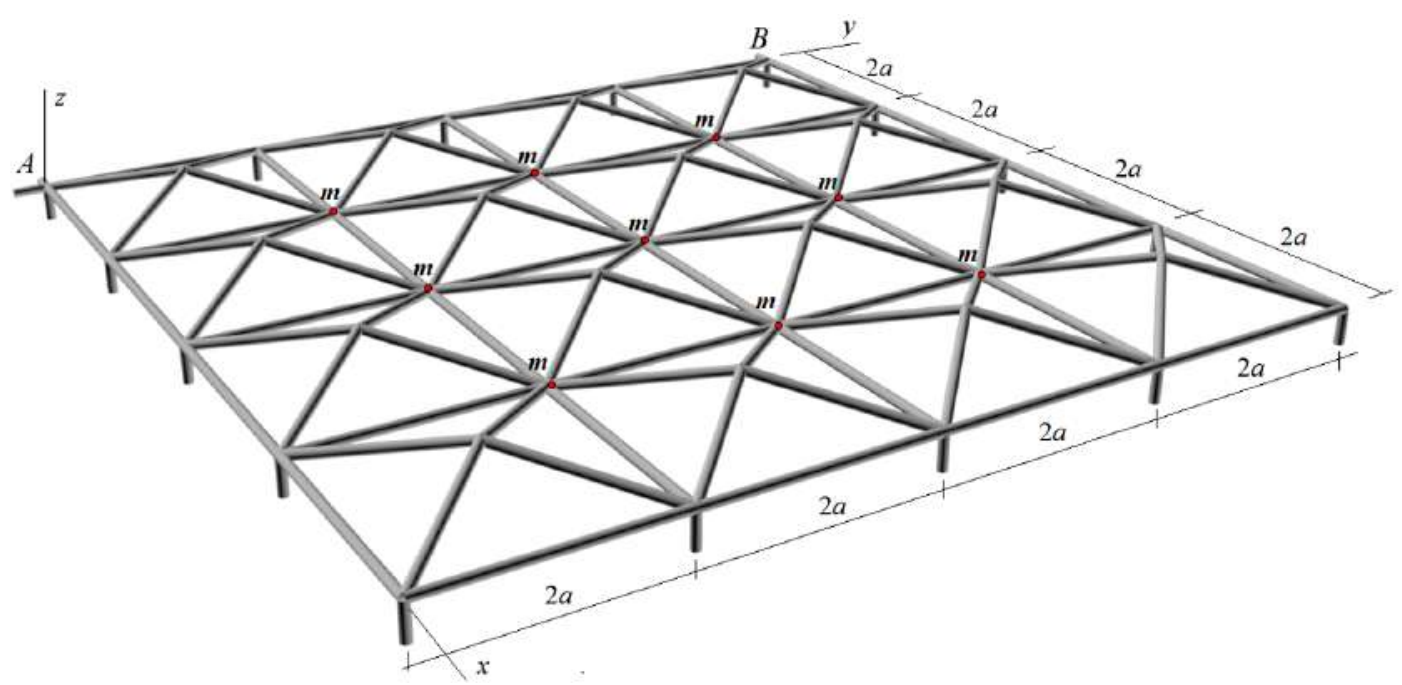

Fig. 1. Girder at $n=4$ under the concentrated load 
In total, the structure contains $r=6 n^{2}+6 n+3$ rods, including $4 n$ vertical support posts and three additional horizontal braces at corners $A$ and $B$. The inertial properties of the structure are modeled by concentrated masses at the nodes of the lower lattice belt. The number of degrees of freedom of the considered model given the fact that the displacement of the weights is considered only along the vertical, is equal to $N=(n-1)^{2}$. The formulas for the static deflection of this structure and the distribution of forces in the rods were obtained in [13]. In order to calculate the rigidity of the truss when calculating the natural frequencies, the scheme for calculating the forces in the rods presented in this study will be used.

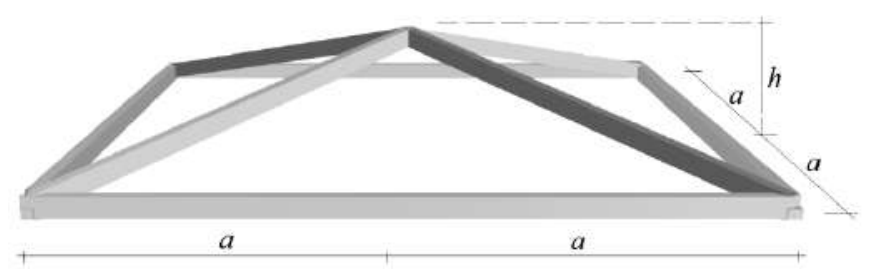

Fig. 2. Element (cell) of the surfacing

2. Donkerley method for identifying the fundamental frequency. The mathematical model of the structure is specified in a coordinate system with the origin at node $A$. The coordinates of the girder hinges are entered into the program for calculating the forces:

$$
\begin{aligned}
& x_{k}=2 a(i-1), y_{k}=2 a(j-1), z_{k}=0, \\
& k=i+(j-1)(n+1), i, j=1, . ., n+1,
\end{aligned}
$$

and the coordinates of the pyramid tops:

$$
\begin{aligned}
& x_{k}=2 a(i-1)+a, y_{k}=2 a(j-1)+a, z_{k}=h, \\
& k=i+(j-1) n+(n+1)^{2}, \quad i, j=1, . ., n .
\end{aligned}
$$

The structure of the bar connections at the nodes is coded by conditional vectors containing the numbers of the ends of the corresponding bars. The matrix of equilibrium equations for nodes of size $r \times r$ consists of the direction cosines of the forces in the rods. The formula for calculating the lower estimate of the first frequency according to Donkerley is ad follows:

$$
\omega_{D}^{-2}=\sum_{k=1}^{N} \omega_{k}^{-2},
$$

where $\omega_{k}$ is the partial frequency. In order to calculate it, the equation of oscillations of one of the system weights is employed:

$$
m \ddot{z}_{k}+d_{k} z_{k}=0, k=1, \ldots, N,
$$


where $d_{k}$ is the coefficient of rigidity of the structure calculated by the value of its deflection from the action of a vertical force on the node $k ; z_{k}$ is the vertical displacement of the mass $m$; $\ddot{z}_{k}$ is the acceleration. Hence we get the partial frequency:

$$
\omega_{k}=\sqrt{d_{k} / m}
$$

Mohr's integral is used to identify the stiffness coefficient:

$$
\delta_{k}=1 / d_{k}=\sum_{\alpha=1}^{r-4 n-3}\left(S_{\alpha}^{(k)}\right)^{2} l_{\alpha} /(E F) .
$$

Here there are the following denotations: $S_{\alpha}{ }^{(k)}$ is the force in the rod $\alpha$ from the action of a single vertical force at the node $k$ where the mass is located; $E F$ is the stiffness of the rods; $1_{\alpha}$ is the length of the rod $\alpha$. The summing in (3) is performed over all the rods, except $4 n$ vertical support posts and three horizontal rods of ties at points $A$ and $B$, which are assumed to be nondeformable. According to (2):

$$
\omega_{D}^{-2}=m \sum_{k=1}^{N} \delta_{k}=m \Delta
$$

where

$$
\Delta=\sum_{\alpha=1}^{r-4 n-3}\left(S_{\alpha}^{(k)}\right)^{2} l_{\alpha} /(E F) .
$$

Calculations of trusses with a different number of panels show that the form of this expression does not depend on the number of panels:

$$
\Delta=\left(C_{1} a^{3}+C_{2} c^{3}\right) /\left(h^{2} E F\right)
$$

where

$$
c=\sqrt{2 a^{2}+h^{2}} \text {. }
$$

The coefficients $C_{1}$ and $C_{2}$ depend on $n$ and are determined by means of induction. Sequential calculation of farms with an increasing number of panels yields a number of coefficients whose common terms are obtained as solutions of recurrent equations and have the form:

$$
\begin{aligned}
& C_{1}=2(n-1)\left(20 n^{5}-4 n^{4}-25 n^{3}+5 n^{2}+5 n-1\right) /(45 n), \\
& C_{2}=\left(n^{2}-1\right)^{2} / 9 .
\end{aligned}
$$

The derivation of these formulas required extraordinarily large expenditures of computer resources and time for such tasks. While obtaining the recurrent equation

$$
C_{1, n}=7 C_{1, n-1}-21 C_{1, n-2}+35 C_{1, n-3}-35 C_{1, n-4}+21 C_{1, n-5}-7 C_{1, n-6}+C_{1, n-7}
$$

for the coefficient $C_{l}$, it was necessary to calculate the frequencies of girders with the number of panels from 2 to 15 . For a sequence of shorter length, the Maple operators do not reveal the recurrence equation. Maple is very slow in performing character conversions. If it took seve- 
ral seconds to calculate the lower bound of the first frequency of a girder with two panels on each side of the surfacing (one degree of freedom) while the result was obtained with $n=14$, the program had been running for more than seven hours. A considerable acceleration of the calculations was obtained by using the axial symmetry of the structure and the inverse matrix method for solving the system of equations for the equilibrium of nodes. The inverse matrix method for solving a system of linear equations turned out to be much faster than the standard LinearSolve operator from the LinearAlgebra package. As a result, first frequency was analytically estimated

$$
\omega_{D}=h \sqrt{E F /\left(C_{1} a^{3}+C_{2} c^{3}\right)}
$$

with the coefficients (5).

3. Numerical solution of the problem on the spectrum of natural frequencies. Let us estimate the error of the obtained solution by comparing it with the numerical one for the minimum frequency of the entire frequency spectrum. The system of differential equations for the dynamics of $N$ loads located at the lattice nodes in matrix form has the form:

$$
M_{N} \ddot{Z}+D_{N} Z=0 \text {, }
$$

where $Z$ is the vector of the vertical displacements of the loads; $D_{N}$ is the stiffness matrix; $M_{N}=m I_{N}$ is the diagonal matrix of the inertia sized $N \times N ; \ddot{Z}$ is the acceleration vector.

The compliance matrix $B_{N}$ is the inverse of the stiffness matrix $D_{N}$ and is calculated by means of Mohr's formula:

$$
b_{i j}=\sum_{\alpha=1}^{r-4 n-3} S_{\alpha}^{(i)} S_{\alpha}^{(j)} l_{\alpha} /(E F)
$$

Multiplying (7) by the matrix $B_{N}$ on the left considering the identity $\ddot{Z}=-w^{2} Z$, the task will be reduced to the problem of own numbers of the matrix $B_{N}: B_{N} Z=\lambda Z$ where $\lambda=1 /\left(m \omega^{2}\right)$ is the own number of the matrix $B_{N}$. At $N>3$ this task is only solved numerically. In order to identify the eigenvalues of the matrix $B_{N}$, the Eigenvalues operator of the Maple system is used. The numerical solution and analytical estimate (6) are compared in the graph (Fig. 3). E.g., the modulus of elasticity of steel $E=2 \times 10^{5} \mathrm{MPa}$ and the cross-sectional area of the rods $F=50 \mathrm{~cm}^{2}$, the masses at the nodes $m=1200 \mathrm{~kg}, a=3 \mathrm{~m}, h=1 \mathrm{~m}$ are taken. The curves converge at the beginning of the graph and with an increase in the number of panels. The maximum discrepancy between the two solutions reaches $45 \%$. Note that Donkerley's estimate for flat girders is a lot more accurate [15]. 


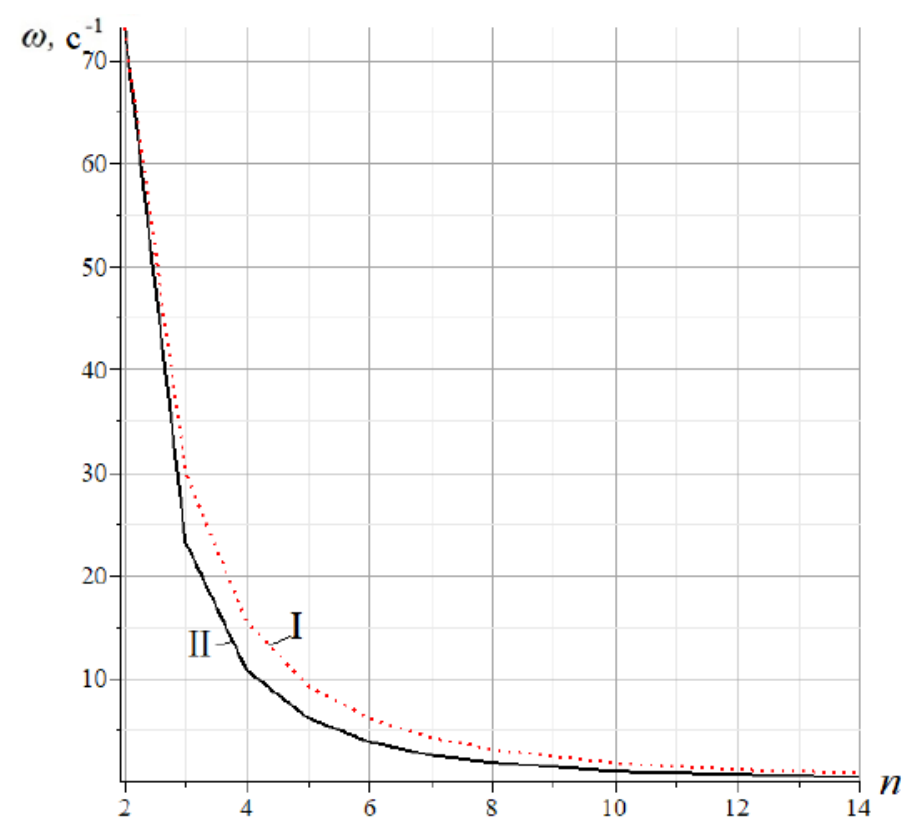

Fig. 3. Major frequency depending on the number of panels: I is the numerical solution; II is the analytical estimate (5)

The dependence of the first frequency on the height of the girder $h$ turns out to be nonlinear. This can be seen in the graph (Fig. 4). At a certain value of $h$, a distinct maximum is observed. The graphs are plotted for $n=4$ and three values of the panel length $a$. The maximum frequency value increases as the panel side size decreases.

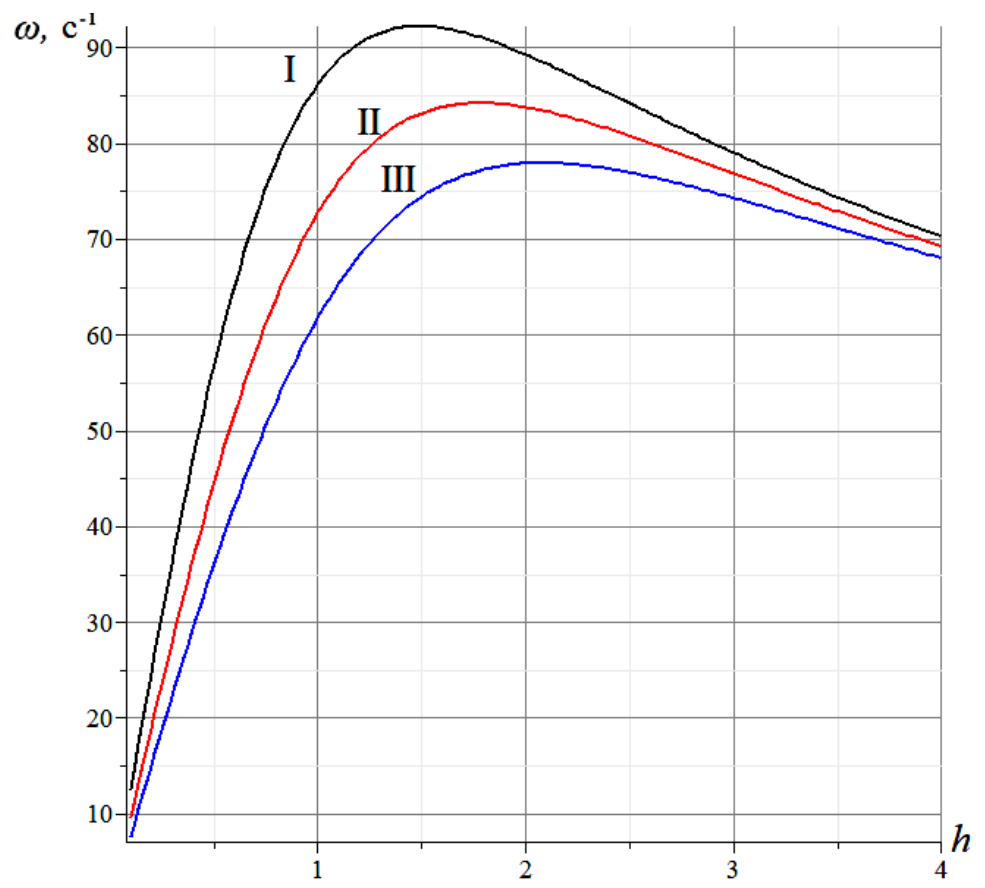

Fig. 4. Dependence of the first frequency on the height of the surfacing:

$$
\mathrm{I}-a=0.5 \mathrm{~m} ; \mathrm{II}-a=0.6 \mathrm{~m} ; \mathrm{III}-a=0.7 \mathrm{~m}
$$


4. The spectrum of natural frequencies. The numerical method enables the entire spectrum of natural frequencies to be calculated. In Fig. 5 spectrum frequencies of each of the girders with the number of panels $n=2, \ldots, 10$ are conventionally united by a broken line. Each point in this polyline is a separate frequency. The spectra were calculated for the same dimensions and masses as the curves in Fig. 3.

The abscissa axis $k$ shows the serial numbers of the frequencies of the spectra ordered in ascending order. For each girder, starting from $n=3$, multiple frequencies ("steps" in the curves) are identified. At the same time, the following law was observed. The number of multiple frequencies for the girders with the panels $n=2,3, \ldots, 11, \ldots$ is $0,1,2,4,6,9,12,16,20,25, \ldots$ The common term of this sequence, expressing the number of multiple frequencies depending on the number of the panels on the sides of the surfacing, is

$$
K(n)=\left(2 n^{2}+(-1)^{n}-1\right) / 8 \text {. }
$$

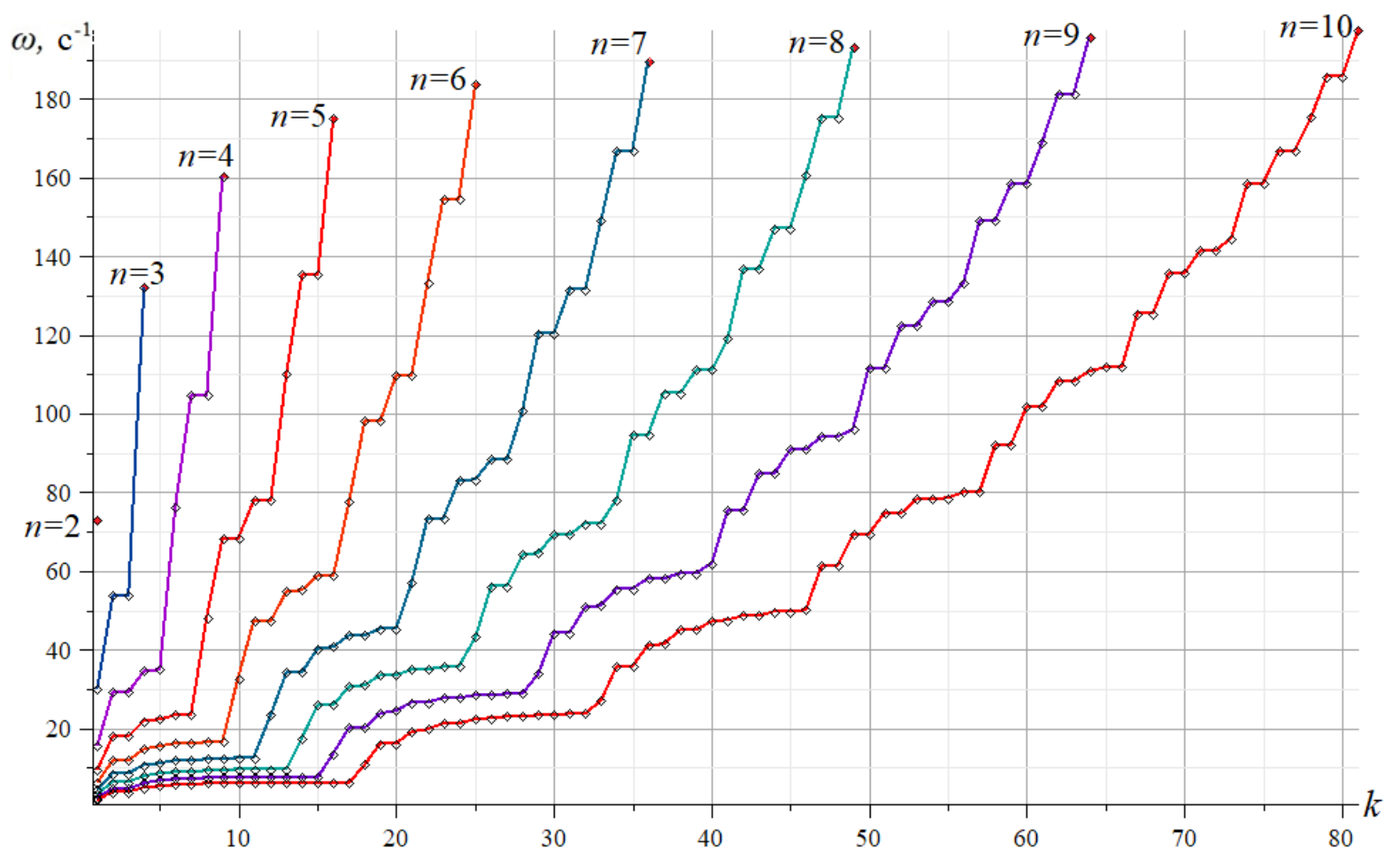

Fig. 5. Spectra of the own frequencies

This function allows one to predict the number of multiple frequencies for a surfacing with an arbitrary, even very large, number of panels with no numerical calculations, i.e., accurately for those cases where the calculated matrices are large and accurate, and the speed of calcula- 
tions decreases. Additionally, the graph shows a monotonic increase in the value of the higher frequencies of the trusses with increasing $n$. The upper frequency increases asymptotically approaching some constant value. Undoubtedly, the higher frequencies of the spectrum are not much in demand in the practice of dynamic calculations as the lower frequencies, however, the knowledge of the upper frequency limit can be instrumental in calculations. According to the constructed picture of the spectra of girders with a different number of panels for an approximate estimate of the higher frequency, one can take the higher frequency at a certain value of $n$ as the upper limit, e.g., at $n=8$, assuming with a high level of confidence that the forecast error of the upper frequency limit at $n>8$ will be small. In the above example, this error is no more than a few percent.

Conclusions. The algorithm for deriving an analytical estimate of the first frequency of natural vibrations of a spatial statically definable girder yields a compact formula which is convenient for calculations with an acceptable level of accuracy, although less than in similar formulas for plane circuits. It is shown that the dependence of the vibration frequency on the height of the structure has an extremum that makes it possible for the designer to choose the optimal dimensions of the structure based on the requirements for the value of the first natural frequency. The structure of the vibration frequency spectra of regular girders reveals a regular arrangement of multiple frequencies. The formula for calculating the number of multiple frequencies is obtained. It is noticed that starting from a certain number of panels, the highest frequency of the spectrum does not depend much on the number of panels. The applied algorithm can be employed for deriving similar estimates for other regular structures.

\section{References}

1. Belyankin N. A., Boiko A. Yu. Formuly dlya progiba balochnoi fermy s proizvol'nym chislom panelei pri ravnomernom zagruzhenii [Formulas for deflection of a beam truss with an arbitrary number of panels at uniform loading]. Stroitel'naya mekhanika i konstruktsii, 2019, no. 1 (20), pp. 21-29.

2. Ovsyannikova V. M. Zavisimost' deformatsii balochnoi fermy trapetsevidnoi formy ot chisla panelei [Dependence of deformations of a trapezoidal beam truss on the number of panels]. Stroitel'naya mekhanika $i$ konstruktsii, 2020, no. 3 (26), pp. 13-20.

3. Terze S. V. Analiticheskii raschet zavisimosti deformatsii konsol'noi stoiki ot chisla panelei v sisteme Maple [Analytical calculation of the dependence of the deformations of the console rack on the number of panels in the Maple system]. Stroitel'naya mekhanika i konstruktsii, 2020, no. 2 (25), pp. 16-24.

4. Abdikarimov R., Khodzhaev D., Vatin N. To Calculation of Rectangular Plates on Periodic Oscillations. MATEC Web of Conferences, 2018, no. 245. doi: 10.1051/matecconf/201824501003. 
5. Al Rjoub Y., Hamad A. Free vibration of axially loaded multi-cracked timoshenko beams. Magazine of Civil Engineering, 2021, no, 8 (100), pp. 10002-10002. doi: 10.18720/MCE.100.2.

6. Bao T., Andrew Swartz R., Vitton S., Sun Y., Zhang C., Liu Z. Critical insights for advanced bridge scour detection using the natural frequency. Journal of Sound and Vibration, 2017, no. 386, pp. $116-133$. doi: 10.1016/j.jsv.2016.06.039.

7. Cao L., Li J., Zheng X., Chen Y. F. Vibration behavior of large span composite steel bar truss-reinforced concrete floor due to human activity. Steel and Composite Structures, 2020, no. 4 (37), pp. $391-404$. doi: $10.12989 /$ scs.2020.37.4.391.

8. Hutchinson R. G., Fleck N. A. Microarchitectured cellular solids - the hunt for statically determinate periodic trusses. ZAMM Z. Angew. Math. Mech., 2005, no. 85, pp. 607-617. doi: https://doi.org/10.1002/ zamm.200410208.

9. Hutchinson R. G., Fleck N. A. The structural performance of the periodic truss. J. Mech. Phys. Solids, 2006, no. 54, pp. 756-782. doi: 10.1016/j.jmps.2005.10.008.

10. Ilyushin A. The formula for calculating the deflection of a compound externally statically indeterminate frame. Structural mechanics and structures, 2019, no. 3 (22), pp. 29-38.

11. Kilikevicius A., Fursenko A., Jurevicius M., Kilikeviciene K., Bureika G. Analysis of parameters of railway bridge vibration caused by moving rail vehicles. Measurement and Control (United Kingdom), 2019, no. 9-10 (52), pp. 1210-1219. doi: 10.1177/0020294019836123.

12. Kirsanov M. N., Tinkov D. V. Analysis of the natural frequencies of oscillations of a planar truss with an arbitrary number of panels. Vestnik MGSU, 2019, no. 4 (14), pp. 284-292. doi: 10.22227/19970935.2019.3.284-292.

13. Kirsanov M. N. Analytical calculation of deformations of a truss for a long span covering. Vestnik MGSU, 2020, no. 10 (15), pp. 1399-1406. doi: 10.22227/1997-0935.2020.10.1399-1406.

14. Kirsanov M. N., Vorobyev O. V. Calculating of a spatial cantilever truss natural vibration frequency with an arbitrary number of panels: analytical solution. Construction of Unique Buildings and Structures, 2021, no. 94, pp. 9402. doi: 10.4123/CUBS.94.2.

15. Kirsanov M. N., Petrichenko E. A., Vorobev O. V. The formula for the lower estimate of the fundamental frequency of natural vibrations of a truss with an arbitrary number of panels. Construction of Unique Buildings and Structures, 2021, no. 1 (94), pp. 9403-9403. doi: 10.4123/CUBS.94.3.

16. Liu M., Cao D., Zhang X., Wei J., Zhu D. Nonlinear dynamic responses of beamlike truss based on the equivalent nonlinear beam model. International Journal of Mechanical Sciences, 2021, no. 194, pp. 106197. doi: 10.1016/j.ijmecsci.2020.106197.

17. Rakhmatulina A. R., Smirnova A. A. Two-parameter derivation of the formula. Postulat, 2018, no. 5-1 (31).

18. Santana M. V. B., Goncalves P. B., Silveira A. M. Closed-form solutions for the symmetric nonlinear free oscillations of pyramidal trusses. Physica D: Nonlinear Phenomena, 2021, no. 417, pp. 132814. doi: 10.1016/j.physd.2020.132814.

19. Tinkov D. V. The optimum geometry of the flat diagonal truss taking into account the linear creep. Magazine of Civil Engineering, 2016, no. 1 (61), pp. 25-32. doi: 10.5862/MCE.61.3.

20. Ufimtcev E. Dynamic Calculation of Nonlinear Oscillations of Flat Trusses. Part 2: Examples of Calculations. Procedia Engineering, 2017, no. 206, pp. 850 - 856. doi: 10.1016/j.proeng.201. 
21. Ufimtsev E., Voronina M. Research of Total Mechanical Energy of Steel Roof Truss during Structurally Nonlinear Oscillations. Procedia Engineering, 2016, no. 150, pp. 1891-1897. doi: 10.1016/j.proeng.2016.07.188.

22. Vatin N. I., J. Havula, L. Martikainen, Sinelnikov A. S., A. V. Orlova, Salamakhin S. V. Thin-walled crosssections and their joints: Tests and FEM-modelling. Advanced Materials Research, 2014, no. 945-949, pp. 1211-1215. doi: 10.4028/www.scientific.net/AMR.945-949.1211.

23. Voropay R. A., Domanov E. V. The dependence of the deflection of a planar beam truss with a complex lattice on the number of panels in the system Maple. Postulat, 2019, no. 1.

24. Voropay R. A., Domanov E. V. The formula for the dependence of the deflection of a truss with an asymmetric lattice on the number of panels. Postulat, 2018, no. 6, p. 61.

25. Voropay R. A. Analytical calculation of the deflection of a beam truss with parallel belts. Postulat, 2018, no. 6 , p. 96 .

26. Voropay R. A. Derivation of the formula for the deflection of the truss with additional horizontal struts. Postulat, 2018, no. 6, p. 105. 